\title{
CALCULATION FOR DEFLECTION OF CONTINUOUS REINFORCED CONCRETE BEAMS IN CONSIDERATION OF MOMENT REDISTRIBUTION
}

\author{
By Dajun DING*
}

\begin{abstract}
In is known that for more precisely calculating the deflection of continuous reinforced concrete beams under service load, the redistribution of internal forces at this stage should be considered. For this purpose, the author suggests two new formulas to calculate the adjusting coefficients of support moments. As establishing the formulas, the translation of inflection points due to moment redistribution has been taken into account. It is reasonable theoretically and can give calculated results in good accordance with tests. In this paper, three diagrams of deflection coefficient are given so as to simplify the calculation greatly. Besides, the consideration of long-term influence on deflection is also proposed.

Keywords : inflection point, adjusting coefficient of moment, short- and long-term stiffness
\end{abstract}

\section{INTRODUCTION}

In the twenties, C. Bach and O. Graf discovered the phenomena of redistribution of internal forces in beams with fixed ends. Soon afterwards, German and English scholars observed also similar facts, which were also confirmed with the tests by Soviet (USSR) Central Academy of Industry Construction Research (ZLIPS-transliteration of Russian).

In the early stage, the redistribution of internal forces in ultimate state was only taken into account in design for calculating the carrying capacity of continuous reinforced concrete slabs and beams. As early as 1941, in the article 701 of ACI Code, the coefficients proposed for designing continuous slabs and beams of two or more approximately equal spans, subjected to uniformly distributed loads were actually given with appropriate consideration of the redistribution of moments. For example, the positive moment at center of interior span was proposed to take (1/16) $q l^{2}$, but from the elastic theory, the positive moment at middle span center of 5-span continuous beam, as considering the unfavorable distribution of live load ${ }^{1)}, M_{3}=$ $(0.085 p+0.046 g) l^{2}=(0.085 \times 3 g+0.046 g) l^{2}=0.301 g l^{2}=(0.301 / 4)(p+g) l^{2}=0.0753 q l^{2}>(1 / 16)$ $q l^{2}$, so it can be seen that the redistribution was properly considered, but it is still safe because the sum of the absolute average support moment and span moment is greater than the span moment of simply supported member. In the above, $p, g$ and $q$ are live, dead and total uniform loads, according to the prescription of ACI Code (1941), $p \leqq 3 g$. In USSR Specification published in 1950, similar considerations were prescribed, but for the negative moment at the face of interior supports, a small value of $(1 / 16) q l^{2}$ was taken, $(1 / 16) q l^{2}+(1 / 16) q l^{2}=(1 / 8) q l^{2}$, i. e. , a full redistribution of moments was considered. As far as the author knows, the earliest formal prescription to consider the redistribution of moments under

* Professor, Department of Civil Engineering, Nanjing Institute of Technology, PRC (Nanjing, Jiangsu, PRC) 
service load in continuous beams was made in USSR Specification published in 1960.

Because, in indeterminate reinforced concrete structures, the redistribution of internal forces occurs from the incipience of loading, but its influence is very small until cracking. As concrete cracks at one or some sections with max. or min. moment, the stiffness of this or these sections decreases largely, the influence of redistribution of internal forces begins to become obvious, that should be considered in calculation. Fig. 1 shows the complete curve of middle-support moment versus load in a continuous reinforced concrete beam of two spans, under a central concentrated load in each span, tested by Doctorial student Mr. Zhang under the guidence of the author ${ }^{2)}$. In this figure, the calculated curve (actually, straight line) following elastic theory is also given. It can be seen that the phenomena of moment redistribution appear obvious after the support section cracked.

For considering the redistribution of internal forces under service load to calculate the deflection of continuous beams (Fig. 2 a)), it is proposed to use the method of conjugate beam, at every interior support of which, a hinge should be inserted as shown in Fig. 2 b), because the hinge can only transfer shear but can't transfer moment, it corresponds to that the interior supports will rotate but no displacement can occur there generally. In the conjugate beam of a 3-span continuous beam shown in Fig. 2 b), there are 2 unknown moments which should be adjusted due to the change of section stiffness, and 4 unknown shears $R_{A}^{c}, \cdots \cdots R_{D}^{c}$, i. e., altogether 6 unknowns. The deformation continuities at interior supports should be kept, which are equivalent to the equalities of shears at two sides of interior hinge, so there are only unknown $R_{B}^{c}$ and another $R_{C}^{c}$. In this case, there will be two statical equilibrium conditions in every span, altogether provide 6 conditions that can give sole solution of the 6 unknowns. When there are $n$ spans, there will be $(n+1)$ unknown shears and $(n-1)$ unknown interior support moments, i. e., altogether $2 n$ unknowns which can be solved with $2 n$ statical conditions. But the solution is very complicated, because the unknowns will be related to the section stiffness which is also concerned with the moments under service load, so it is necessary to make repeatedly trial computations. Generally, in the case of continuous beams with equal and approximate equal spans (difference $\leqq 20 \%$ ), the rotations of the interior supports aren't large. For simplicity, the interior supports can be considered as fixed, therefore, the interior spans will be transformed into the beams with two ends fixed and the external spans into the beams with one end simply supported (following the original cases) and another fixed, of which the conjugate beams will become of those as shown in Fig. 2c), i. e., the sections of interior supports are cut out so as to become into free ends. Every span will be in self-equilibrium under the load of corresponding $M / B$, i. e., the calculated reaction (shear) at the free end should be equal to zero and the interior support moments of real beam will be slightly changed from $M$ to $M^{\prime}$. It should be pointed out that these conjugate beams cut out will be unstable under another loads.

For regarding the redistribution of internal forces (moments) in continuous reinforced concrete beams at service stage following the above simplified sketch, some calculation proposals have been suggested internationally. The method of adjusting coefficients of support moment in USSR Specification is the

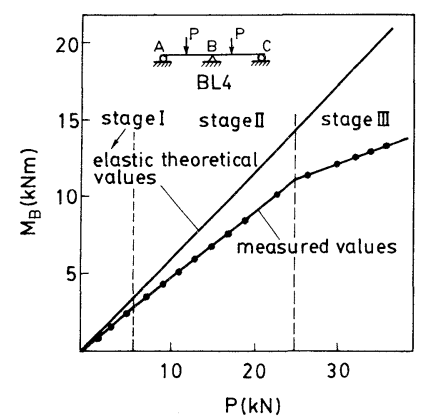

Fig. 1 Complete curve of load-support moment. a)

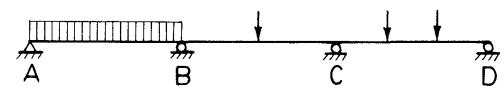

b)

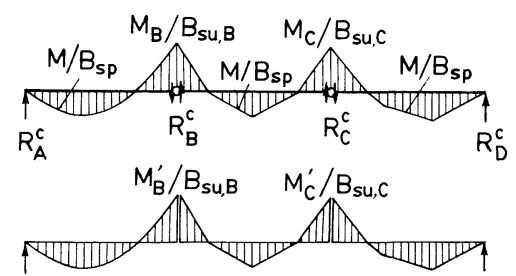

Fig. 2 Continuous beam and its conjugate beams. 
simplest one. But, as the variation of the ratio of span and support stiffness is large, USSR coefficients will give too large adjustment of support moments as the actual. In 1964, other formulas of adjusting coefficient, put forward by the author, can give results better agreeable to the actual ones, including the cases of large variation of stiffness ${ }^{3)}$.

\section{ADJUSTMENT OF SUPPORT MOMENT IN CONTINUOUS REINFORCED CON- CRETE BEAMS}

\section{(1) Calculation formulas}

a) USSR formulas

Adjusting coefficient $\mu$ of support moment are proposed as follows

for interior span : $\mu=\frac{2}{1+\beta}$

for external span : $\mu=\frac{1.5}{0.5+\beta}$

where $\beta=B_{s p} / B_{s u} ; B_{s p}, B_{s u}$ are the section stiffness of span and support, respectively.

b) Author's proposals ${ }^{4)}$

As mentioned above, at the service stage after crack (s) occurs (occur), the obvious redistribution of moments in span and at support will appear, thus the position of inflection point will change from that determined following elastic theory. Fig. 3 shows the measured results from the test of continuous fly-ash ceramsite concrete beams conducted by Master student Mr. Wu Zhishen under the guidance of the author. Therefore, this characteristic should be considered in the determination of coefficient $\mu$.

The calculation of deflection is conducted according to the principle of min. stiffness ${ }^{4}$, i. e., the curvatures $M / B$ in the region of positive moment are determined according to the stiffness of the section with max. span moment, and those in the region of negative moment according to the stiffness of the section with min. support moment, respectively. Though, only from the view of subjecting to normal bending, the calculation following the principle of min. stiffness will give conservative (on large side) results, yet, it can still give results approaching to the actual because the increase influence on deflection due to shear deformation and oblique bending hasn't been taken into account, and indeed, this influence exists after cracking $^{4) .5)}$.

The further discussion on the principle of min. stiffness will be given in APPENDIX.

Now, it will turn to determining the adjusting coefficient $\mu$. The interior span will be calculated as a

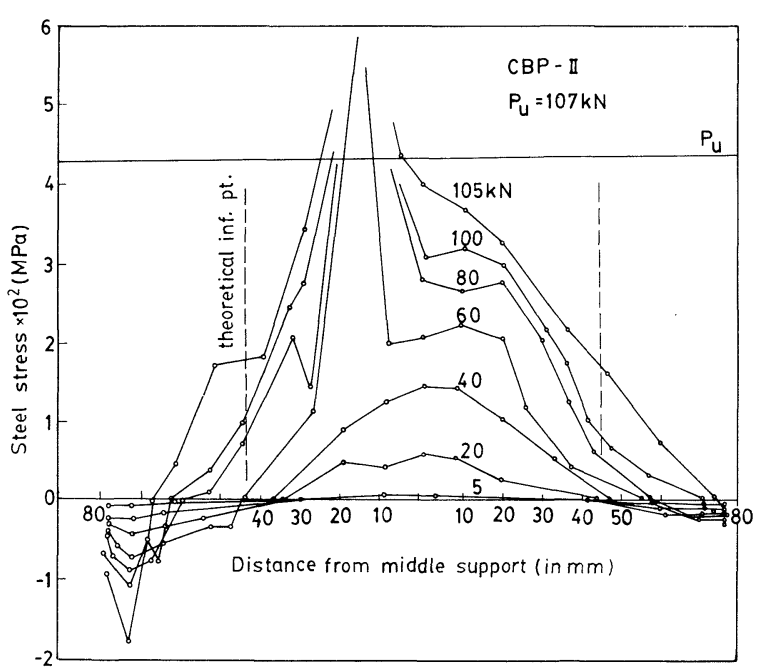

Fig. 3 Change of position of inflection points. a)

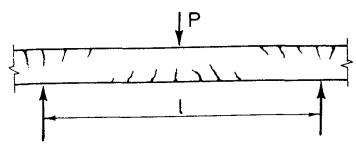

b)

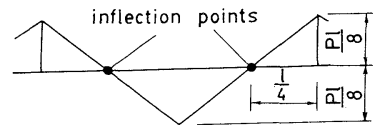

c)

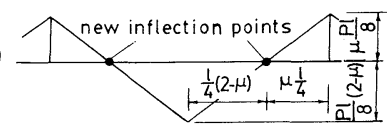

d)

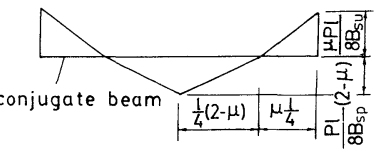

Fig. 4 Calculation of internal span as fixed beam. 
beam fixed at both ends as discussed in the above. Fig. 4 a) shows the interior span of actual beam after cracking under service load; Fig. 4 b) - the moment diagram following elastic theory; Fig. 4 c) - the moment diagram with new inflection points due to the redistribution of internal forces; Fig. $4 \mathrm{~d}$ ) conjugate beam of a fixed end beam with load $M / B_{s p}$ in the region of positive moment and $M / B_{s u}$ in that of negative one following the principle of min. stiffness. In Fig. $4 \mathrm{~d}$ ), there will be an equality, i. e., the area of positive $M / B_{s p}$ should be equal to that of negative $M / B_{s u}$ due to zero shear at the supports of conjugate beam or zero rotation at those of actual beam, then

$$
\frac{1}{2} \frac{P l}{8 B_{s p}}(2-\mu) \frac{l}{4}(2-\mu)=\frac{1}{2} \frac{\mu P l}{8 B_{s u}} \frac{\mu l}{4}
$$

simplifying gives

$$
(2-\mu)^{2}=\mu^{2} \frac{B_{s p}}{B_{s u}}=\mu^{2} \beta
$$

therefore, the adjusting coefficient can be found as follows:

$$
\mu=\frac{2}{1+\sqrt{\beta}}
$$

The external span will be calculated as a beam with one simply supported end and one fixed end. For this case, an approximate simplification should be made to the calculated results, the final result will be obtained as follows ${ }^{6)}$

$$
\mu=\frac{1.5}{0.5+\sqrt{\beta}} \text {. }
$$

\section{( 2) Calculation of stiffness}

The section stiffness under short-term loading may be calculated following the formula proposed by the author and adopted by China Design Code (TJ 10-74) ${ }^{7)}$

$$
B_{s}=\frac{E_{s} A_{s} d^{2}}{1.15 \psi+\frac{0.2+6 \alpha_{E} \rho}{1+2 \gamma^{\prime}}}
$$

where $E_{s}, A_{s}$ : elasticity modulus and section area of tensile bars;

$\rho:$ steel ratio, $\rho=A_{s} / b_{w} d$

$b_{w}, d:$ web width and effective depth of section;

$E_{c}$ : elasticity modulus of concrete;

$\alpha_{E}=E_{s} / E_{c}:$ ratio of elasticity moduli of steel and concrete ;

$\gamma^{\prime}$ : strengthening (with respect to $\left.b_{w} d\right)$ coefficient of compressive flange, $\gamma^{\prime}=\left(b-b_{w}\right) t$ $/ b_{w} d, b, t:$ width and thickness of compressive flange ;

$\psi:$ nonuniformity coefficient of tensile steel strains (considering tension stiffening influence of tensile concrete) :

$$
\psi=1.2\left[1-\frac{0.235\left(1+2 \gamma_{1}+0.4 \gamma_{1}^{\prime}\right) b_{w} h^{2} f_{c t}}{M}\right] \geqq 0.4, \leqq 1.0
$$

$\gamma_{1}, \gamma_{1}^{\prime}$ : strengthening (with respect to $b_{w} h$ ) coefficients of tensile and compressive flanges ; $\gamma_{1}=$ $\left(b_{t}-b_{w}\right) t_{t} / b_{w} h ; \gamma_{1}^{\prime}=\left(b-b_{w}\right) t / b_{w} h$

$b_{t}, t_{t}:$ width and thickness of tensile flange; $h:$ overall depth of section;

$f_{c t}$ : average tensile strength of concrete.

Following the principle of min. stiffness, checking the measured deflections of 197 simply supported beams tested in China gives the average $\delta_{m} / \delta=0.963\left(\delta_{m}, \delta:\right.$ measured and calculated deflections $)$, coefficient of deviation $C_{v}=0.117$; checking the data of 139 beams tested abroad gives the average $\delta_{m} / \delta=$ 1. 003 , coefficient of deviation $C_{v}=0.1435$. It can be seen that Eqs. ( 5 ), (6) can give exact calculated stiffness. But checking the above data by using the formulas in CEB-FIP Model Code (1978) and ACI Code (318-83) gives respectively : average $\delta_{m} / \delta=1.206, C_{v}=0.1896$ and average $\delta_{m} / \delta=1.003, C_{v}=$ 
Table 1 Comparison of calculated moments with measured moments.

\begin{tabular}{|c|c|c|c|c|c|c|c|}
\hline \multirow{2}{*}{$\begin{array}{l}\text { Data } \\
\text { from }\end{array}$} & \multirow{2}{*}{$\begin{array}{l}\text { Number } \\
\text { of } \\
\text { tested } \\
\text { beams }\end{array}$} & \multicolumn{2}{|c|}{$\begin{array}{c}\text { M calculated } \\
\text { following } \\
\text { elastic theory }\end{array}$} & \multicolumn{2}{|c|}{$\begin{array}{l}\text { M calculated } \\
\text { following } \\
\text { Eq. (2) }\end{array}$} & \multicolumn{2}{|c|}{$\begin{array}{c}\text { M calculated } \\
\text { following } \\
\text { Eq. (4) }\end{array}$} \\
\hline & & $\begin{array}{c}\text { aver. } \\
\frac{M_{1}^{m}-M_{1}}{M_{1}} \\
\%\end{array}$ & $\begin{array}{c}\text { aver. } \\
\frac{M_{B}^{m}-M_{B}}{{ }_{B}} \\
\not 6\end{array}$ & $\frac{\frac{M_{1}^{m}-M_{1}}{M_{1}}}{\not}$ & $\frac{\frac{M_{B}^{m}-M_{B}}{M_{B}}}{\%}$ & $\frac{\mathrm{M}_{1}^{\mathrm{m}}-\mathrm{M}_{1}}{\mathrm{M}_{1}}$ & $\frac{\mathrm{M}_{\mathrm{B}}^{\mathrm{m}}-\mathrm{M}_{\mathrm{B}}}{{ }_{\mathrm{B}}^{\mathrm{M}_{\mathrm{B}}}}$ \\
\hline $\begin{array}{l}\text { NIT [8] } \\
\text { ABR [9] } \\
\text { QHU [1 } 0]\end{array}$ & $\begin{array}{l}9 \\
8 \\
6\end{array}$ & $\begin{array}{r}7.7 \\
10.8 \\
3.5\end{array}$ & $\begin{array}{r}18.1 \\
13.8 \\
6.9\end{array}$ & $\begin{array}{l}6.7 \\
5.4 \\
6.8\end{array}$ & $\begin{array}{r}14 \cdot 2 \\
6.1 \\
12.9\end{array}$ & $\begin{array}{l}3.7 \\
6.3 \\
2.4\end{array}$ & $\begin{array}{l}8.3 \\
6.6 \\
4.6\end{array}$ \\
\hline Total & 23 & 6.9 & 13.7 & 6.3 & 11.0 & 4.2 & 6.7 \\
\hline
\end{tabular}

0.3304 as well as average $\delta_{m} / \delta=1.068, \quad C_{v}=0.1990$ and $\delta_{m} / \delta=1.1036, C_{v}=0.2603^{4)}$.

\section{( 3 ) Comparison of moment calculation with tests}

The comparison of calculated span and support moments $M_{1}$ and $M_{B}$ with tested moments $M_{1}^{m}$ and $M_{B}^{m}$, measured on two-span continuous beams subjected to symmetrical loads and conducted in China, has been made and the results ${ }^{2)}$ are given in Table 1.

As adding 4 beams tested at $\mathrm{NIT}^{2)}$, the comparison result following Eq. ( 2 ) gives aver. $M_{B}^{m} / M_{B}=1.03$, mean square deviation $\sigma=0.13$, coefficient of deviation $C_{v}=0.13$; following Eq. (4), aver. $M_{B}^{m} / M_{B}=$ $0.96, \sigma=0.07, \quad C_{v}=0.07$.

It can be seen that Eq. (4) proposed by the author can give calculated results in good accordance with tests. Checking the deflections of 9 continuous reinforced concrete beams tested by Dr. Zhang ${ }^{8)}$ at NIT gives average $\delta_{m} / \delta=1.050, C_{v}=0.044$, except for two beams yielded at service stage, of which $\delta_{m} / \delta=$ 1.111 and $1.124, \delta_{m} / \delta$ of the others $=0.972-1.076$. It can be seen that the results calculated following the method proposed in this paper are exact enough. Because $\mu$ must be found following stiffness ratio $\beta$ and the stiffness is related to $M$ which will be varied due to $\mu$, so $\mu$ has to be determined by trial and error, but it is easy to obtain the final result.

\section{(4) Calculation of deflection in continuous beams}

According to the principle of min. stiffness, the calculation of deflection can be conducted by Conjugate Beam Method. Firstly, a simpler case of a beam fixed at both ends and subjected to a central concentrated load will be taken as an example. From the conjugate beam shown in Fig. 4, the distance between the application points of the resultants of two equal triangular loads of $\frac{1}{2} \frac{\mu P l}{8 B_{s u}} \mu \frac{l}{4}=\frac{1}{2} \frac{P l}{8 B_{s p}}(2-\mu) \frac{l}{4}(2-$ $\mu)$ is equal to $\frac{2}{3} \frac{l}{4} \mu+\frac{2}{3} \frac{l}{4}(2-\mu)=\frac{l}{3}$, then the calculation formula of max. (central) deflection can be found from the span moment at center of the conjugate beam as follows:

$$
\begin{aligned}
& \delta_{\max }=\frac{1}{2} \frac{\mu P l}{8 B_{s u}} \mu \frac{l}{4} \frac{l}{3}=\frac{\mu^{2} P l^{3}}{192 B_{s u}} . \\
& =\frac{\beta \mu^{2} P l^{3}}{192 B_{s p}} \text {. }
\end{aligned}
$$

\section{COEFFICIENTS OF DEFLECTION}

\section{(1) Calculation formulas using deflection coefficient}

For simplifying the calculation, the diagrams of max. deflection coefficients can be drawn.

The calculation formulas of $\mathrm{Eq} .(7 \cdot \mathrm{a})$ can be expressed as follows :

$\delta_{\max }=\gamma \frac{P l^{3}}{12 B_{s p}}$ 
where $\gamma$ is the function of $\beta$ and $\mu$, i. e., of $\mu$, because as $\beta$ is given, $\mu$ can be calculated from Eq. (3).

The deflections under the other cases of loading can be calculated by using the following formula : for concentrated load at two third points :

$\delta_{\max }=\gamma \frac{P l^{3}}{6 B_{s p}}$.

for uniform load,

$\delta_{\max }=\gamma \frac{q l^{4}}{24 B_{s p}}$.

If the symmetrical concentrated loads on one span are more than two, the error due to calculating following equivalent uniform load will be small.

(2) Determination of coefficient $\gamma$ for other case

For an example, the coefficient $\gamma$ of external span in continuous beam under uniform load is taken. As the simplified assumption made above is adopted, the calculation will be conducted following the sketch of one end simply supported and the other fixed (see Fig. 2). The reaction of the simply supported end :

$$
R_{A}=\frac{q l}{2}-\frac{\mu q l}{8}=\frac{q l}{2}\left(1-\frac{\mu}{4}\right)
$$

Moment in beam :

$$
M_{x}=\frac{q l}{2}\left(1-\frac{\mu}{4}\right) x-\frac{q x^{2}}{2} .
$$

where $x$ : from the simply supported end.

The position of inflection point from the simply supported end with distance :

$$
a=l\left(1-\frac{\mu}{4}\right)
$$

The reaction of the conjugate beam at the simply supported end :

$$
\begin{aligned}
& R_{A}^{c}=\frac{1}{l}\left\{\frac{\int_{0}^{a}\left[\frac{q l}{2}\left(1-\frac{\mu}{4}\right) x-\frac{q x^{2}}{2}\right](l-x) d x}{B_{s p}}+\frac{\int_{a}^{l}\left[\frac{q l}{2}\left(1-\frac{\mu}{4}\right) x-\frac{q x^{2}}{2}\right](l-x) d x}{B_{s u}}\right\} \\
& =\frac{q l^{3}}{B_{s p}}\left\{\left(1-\frac{\mu}{4}\right)^{3}\left(\frac{1}{24}+\frac{\mu}{96}\right)+\beta\left[\left(\frac{1}{24}-\frac{\mu}{48}\right)-\left(1-\frac{\mu}{4}\right)^{3}\left(\frac{1}{24}+\frac{\mu}{96}\right)\right]\right\} \\
& =\frac{q l^{3}}{B_{s p}}\left\{A+\beta\left[\left(\frac{1}{24}-\frac{\mu}{48}\right)-A\right]\right\}
\end{aligned}
$$

where $A=\left(1-\frac{\mu}{4}\right)^{3}\left(\frac{1}{24}+\frac{\mu}{96}\right)$.

$\delta_{\max }$ occurs at the position where the shear of conjugate beam is equal to zero, i. e.,

$$
R_{A}^{c}-\frac{\int_{0}^{x_{0}}\left[\frac{q l}{2}\left(1-\frac{\mu}{4}\right) x-\frac{q x^{2}}{2}\right] d x}{B_{s p}}=0
$$

i. e. ,

$$
A+\beta\left[\left(\frac{1}{24}-\frac{\mu}{48}\right)-A\right]-\frac{1}{4}(1-\mu) \xi_{0}^{2}+\frac{1}{6} \xi_{0}^{3}=0 .
$$

where $\xi_{0}=x_{0} / l, x_{0}$ : distance from the position of $\delta_{\max }$ to the simply supported end.

$$
\begin{aligned}
\delta_{\max } & =R_{A}^{c} x_{0}-\frac{\int_{0}^{x_{0}}\left[\frac{q l}{2}\left(1-\frac{\mu}{4}\right) x-\frac{q x^{2}}{2}\right]\left(x_{0}-x\right) d x}{B_{s p}} \\
& =\frac{q l^{4}}{B_{s p}}\left\{A+\beta\left[\left(\frac{1}{24}-\frac{\mu}{48}\right)-A\right] \xi_{0}-\frac{1}{12}\left(1-\frac{\mu}{4}\right) \xi_{0}^{3}+\frac{\xi_{0}^{4}}{24}\right\} .
\end{aligned}
$$

as $\mu=1$, then $\beta=1$ and $A=0.021973$, from (e),

$0.021973+[0.020833-0.021973]-0.1875 \xi_{0}^{2}+\frac{1}{6} \xi_{0}^{3}=0$ 
solving by trial and error gives $\xi_{0}=0.422$, then from (f),

$$
\delta_{\max }=\frac{q l^{4}}{B_{s p}}\left\{0.020833 \times 0.422-\frac{1}{12}\left(1-\frac{1}{4}\right) \times 0.422^{3}+\frac{0.422^{4}}{24}\right\}=0.00542 \frac{q l^{4}}{B_{s p}},
$$

it is the same as given following elastical theory, the corresponding $\gamma=24 \times 0.00542=0.13008$.

If the calculation for $\delta_{0}$ at the center of span is made, then

$$
\delta_{0}=\frac{q l^{4}}{2 B_{s p}}\left[A+\left(\frac{1}{24}-\frac{\mu}{48}-A\right)-\frac{3-\mu}{192}\right]
$$

As $\mu=1, \beta=1$, then

$$
\delta_{0}=\frac{q l^{4}}{2 B_{s p}}\left(0.020833-\frac{3-1}{192}\right)=0.0052082 \frac{q l^{4}}{B_{s p}}
$$

$\gamma_{0}=0.125$ is the same as determined following elastical theory.

As comparison with $\gamma$, the error $=-3.9 \%$, it is allowable in practical design. If there aren't diagrams of $\gamma$, it is easier to find $\gamma_{0}$ by computation.

As $\mu=0.874$, then $\beta=\left(\frac{1.5}{\mu}-0.5\right)^{2}=\left(\frac{1.5}{0.874}-0.5\right)^{2}=1.4793$

and

$$
\begin{aligned}
A= & \left(1-\frac{0.874}{4}\right)^{3}\left(\frac{1}{24}+\frac{0.874}{96}\right)=0.024233, \text { from }(\mathrm{e}), \\
& 0.024233+1.4793\left[\frac{1}{24}-\frac{0.874}{48}-0.024233\right]-\frac{1}{4}\left(1-\frac{0.874}{4}\right) \xi_{0}^{2}+\frac{1}{6} \xi_{0}^{3} \\
= & 0.023087-0.195375 \xi_{0}^{2}+\frac{1}{6} \xi_{0}^{3}=0, \quad \xi_{0}=0.434, \\
\delta_{\max }= & \frac{q l^{4}}{B_{s p}}\left\{0.023087 \times 0.434-\frac{1}{12}\left(1-\frac{0.874}{4}\right) 0.434^{3}+\frac{0.434^{4}}{24}\right\} \\
= & 0.00618 \frac{q l^{4}}{B_{s p}}, \text { the corresponding } \gamma=24 \times 0.00618=0.1482 .
\end{aligned}
$$

From Fig. 6, $\gamma=0.148$, it can be seen that the diagram of $\gamma$ is exact enough.

Checking the fixation of the interior support : as $\mu=0.874, a=0.7815 l, R_{A}^{c}=0.023087 q l^{3} / B_{s p}$, then

$$
\begin{aligned}
R_{B}^{c} & =R_{A}^{c}-\frac{\int_{0}^{0.7815 l}\left[\frac{q l}{2}\left(1-\frac{0.874}{4}\right) x-\frac{q x^{2}}{2}\right] d x}{B_{s p}}-\frac{\int_{0.7815 l}^{l}\left[\frac{q l}{2}\left(1-\frac{0.874}{4}\right) x-\frac{q x^{2}}{2}\right] d x}{B_{s u}} \\
& =0.023087 \frac{q l^{3}}{B_{s p}}-\frac{\int_{0}^{0.7815 l}\left(0.39075 q l x-0.5 q x^{2}\right) d x}{B_{s p}}-\beta \frac{\int_{0.7815 l}^{l}\left(0.39075 q l x-0.5 q x^{2}\right) d x}{B_{s p}} \\
& =\frac{q l^{3}}{B_{s p}}(0.023087-0.039775+0.016371)=0.000317 \frac{q l^{3}}{B_{s p}} \ll 0.023087 \frac{q l^{3}}{B_{s p}}
\end{aligned}
$$

it is not equal to zero due to the approximation of Eq. (4), which is derived from beam subjected to a central concentrated load and simplified finally, but is very small as comparison with $R_{A}^{c}$, so can be neglected.

For the above three cases of loading, the diagrams of coefficients $\gamma$ for interior and external spans are given in Fig. 5 7 respectively.

The reader may raise a question that must be clarified. From Eqs. $(7 \cdot a)$ and ( 8$)$, as $\mu \rightarrow 0$, whether $\gamma$ approaches also to 0 ? The answer is negative. Because in this case, $\mu \rightarrow 0$, the corresponding $\beta \rightarrow \infty$, then $\mu^{2} \beta$ is indefinite, so its limiting value should be found in usual way, i. e., $\lim _{\beta \rightarrow \infty} \mu^{2} \beta=\lim _{\beta \rightarrow \infty} \frac{4}{(1+\sqrt{\beta})^{2}}=$ $\lim _{\beta \rightarrow \infty} \frac{4}{(1 / \sqrt{\beta}+1)^{2}}=4$, so $\gamma=4 / 16=0.25$. In the other two cases, the corresponding $\gamma=0.213$ and 0.3125 respectively. 


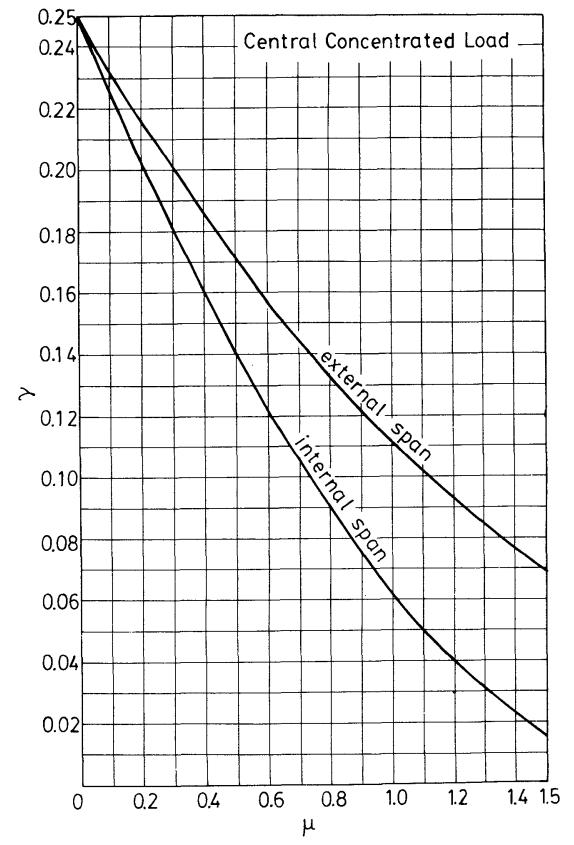

Fig. 5 Diagram of $\mu-\gamma$ in the case of central concentrated load.

\section{CONSIDERATION OF INFLUENCE UNDER LONG- TERM LOADING}

In long-term procedure, it is known that there will produce shrinkage and creep in concrete. Both of these influences will increase the deflection of beams. Besides that the creep occurring in compressive zone will increase the compressive strains and then induce additional deflection, the creep of slip between concrete and steel will cause the increase of tensile steel strains between cracks and will also increase the deflection.

For considering the long-term influence on deflection of beams, the author proposed to calculate the long-term stiffness $B_{l}$ of beam section as follows ${ }^{11)}$ :

$$
B_{l}=\frac{E_{s} A_{s} d^{2}}{1.15 \phi_{l}+\frac{0.2+6 \alpha_{E} \rho}{1+2 \gamma^{\prime}}(1+m \varphi)+\frac{S \varepsilon_{s h}}{0.87 \varepsilon_{s}}}
$$

where

$$
\psi_{l}=1.2\left[1-\left(1-\frac{1}{2} m\right) \frac{0.235\left(1+2 \gamma_{1}+0.4 \gamma_{1}^{\prime}\right) b_{w} h^{2} f_{c t}}{M}\right] \geqq 0.4, \leqq 1.0
$$

$m M_{l} / M, M=M_{s}+M_{l}, M_{s}$ and $M_{l}$ are moments due to short- and long-term loads respectively;

$\varphi:$ creep coefficient ;

$\varepsilon_{s h}:$ shrinkage coefficient ;

$S:$ influence coefficient of restraint of tension steel to shrinkage strain so that there produces deformation difference at top of beam and at steel level thus results in warping: 
$S=0.5+25 \rho \leqq 1.0$

$\varepsilon_{s}:$ calculated strain of tensile steel, $\varepsilon_{s}=M / A_{s} 0.87 d E_{s}$.

It is proposed to take $\varphi=2$ and $\varepsilon_{s h}=4.5 \times 10^{-4}$ under normal conditions of humidity. $\varphi$ and $\varepsilon_{s h}$ should be increased or decreased respectively as the age of concrete at incipience of loading is less than 21 days or more than 40 days. $\varphi$ and $\varepsilon_{s h}$ should also be decreased if the section is doubly reinforced ${ }^{11}$.

The computed comparison of a large number (173 beams) of simply supported beams tested under long-term loading in China and abroad following Eqs. (11) (13) gives aver. $\delta_{l}^{m} / \delta_{l}=0.998, \sigma=0.1508$, $C_{v}=0.1515^{11)}$. Comparing the results of 9 continuous beams under uniform load, tested by G. W. Washa, et al. with those calculated by the proposals in this paper gives $\delta_{l}^{m} / \delta_{l}=0.840 \sim 0.971$, the aver. $=0.905$, $\sigma=0.047, C_{v}=0.052$. It can be seen that the author's proposal for calculating long-term deflection can also give satisfactory results.

\section{EXAMPLE OF CALCULATION}

A monolithic floor with beam spacing $=2.2 \mathrm{~m}$, under uniform load $q=p+g=6.6+14.13=$ $20.73 \mathrm{kN} / \mathrm{m}$, the thickness of slab $=80 \mathrm{~mm}$, width and depth of beam $=200$ and $450 \mathrm{~mm}, d=415$ and $395 \mathrm{~mm}$ in span and at support; the calculated span length $l$ of the external span in the 5 -span continuous beam $=5.33 \mathrm{~m}$; the reinforcements $A_{s}$ in span $=534 \mathrm{~mm}^{2}$, at support $=647 \mathrm{~mm}^{2} ; E_{c}=2.3 \times 10^{4} \mathrm{MPa}, f_{c t}$ $=1.3 \mathrm{MPa}$ and $E_{s}=2.0 \times 10^{5} \mathrm{MPa}$; a half of live load is applied in long-term time, i. e. , $m=(3.3+$ 14. 13) $/ 20.73=0.841$.

According to the current Chinese Design Code (TJ 10-74) ${ }^{7}$, the smallest of the following values should be taken as the calculated flange width of inverted T section at interior support : (1/6) $l$, spacing of beams and $b_{w}+5$ times of slab thickness, among which the last one is smallest, i. e. , $b_{t}=200+5 \times 80=600 \mathrm{~mm}$; for calculating the $\mathrm{T}$ beam (in span), $b=200+12 \times 80=1160 \mathrm{~mm}$.

According to Chinese Code (TJ 10-74) ${ }^{7}$, the cracking moment may be determined through multiplying the elastic value by a coefficient greater than 1 to consider the plasticity of tensile concrete, in this case $\left(b_{t} / b_{w}>2, t_{t} / h<0.2\right)$, this coefficient $=1.5$, the section modulus against tension of the support section, $w_{t}=2.619 \times 10^{9} / 155.8=1.681 \times 10^{7} \mathrm{~mm}^{3}, \quad$ then $M_{c r}=1.5 \times 1.3 \times 1.681 \times 10^{7}=3.278 \times 10^{7} \mathrm{Nmm}=32.78$ $\mathrm{kNm}$.

Assuming $\mu=0.8$, then after adjusting, support moment $=0.8 \times(20.73 / 8) \times 5.33^{2}=58.89 \mathrm{kNm}>$ $32.78 \mathrm{kNm}$, so the support section cracks following calculation and its stiffness should be determined from Eq. ( 5 ) or Eq. (11).

span center moment $=(20.73 / 8) \times 5.33^{2}-(58.89 / 2)=44.16 \mathrm{kNm}$, calculation coefficients for determining stiffness

$$
\begin{aligned}
& \gamma_{1}=\frac{(600-200) \times 80}{200 \times 450}=0.356 \\
& \gamma_{1}^{\prime}=\frac{(1160-200) \times 80}{200 \times 450}=0.853 \\
& \gamma^{\prime}=\frac{(1160-200) \times 80}{200 \times 415}=0.925
\end{aligned}
$$

Stiffness of support section (invertedd $\mathrm{T}$ ) :

$$
\begin{aligned}
& \psi_{l}=1.2\left[1-\left(1-\frac{0.841}{2}\right) \frac{0.235(1+2 \times 0.356) \times 200 \times 450^{2} \times 1.3}{58.89 \times 10^{6}}\right]=0.950 \\
& \alpha_{E} \rho=\frac{20}{2.3} \times \frac{647}{200 \times 395}=8.7 \times 0.00819=0.0713 \\
& S=0.5+25 \times 0.00819=0.705 \\
& \varepsilon_{S}=\frac{58.89 \times 10^{6}}{647 \times 0.87 \times 395 \times 2 \times 10^{5}}=0.00132
\end{aligned}
$$




$$
\begin{aligned}
B_{\text {su }, l} & =\frac{2.0 \times 10^{5} \times 647 \times 395^{2}}{1.15 \times 0.950+(1+0.841 \times 2)(0.2+6 \times 0.0713)+\frac{0.705 \times 450 \times 10^{-4}}{0.87 \times 1.32 \times 10^{-3}}} \\
& =6.614 \times 10^{12} \mathrm{Nmm}^{2}=6.614 \times 10^{3} \mathrm{kNm}^{2}
\end{aligned}
$$

Stiffness of span section ( $\mathrm{T}$ section) :

$$
\begin{aligned}
\phi_{l}= & 1.2\left[1-\left(1-\frac{0.841}{2}\right) \frac{0.235(1+0.4 \times 0.853) \times 200 \times 450^{2} \times 1.3}{44.16 \times 10^{6}}\right]=0.939 \\
\alpha_{E} \rho & =\frac{20}{2.3} \times \frac{534}{200 \times 415}=8.7 \times 0.00643=0.056 \\
S= & 0.5+25 \times 0.00643=0.661 \\
\varepsilon_{s}= & \frac{44.16 \times 10^{6}}{534 \times 0.87 \times 415 \times 2 \times 10^{5}}=1.145 \times 10^{-3} \\
B_{s p, l} & =\frac{2 \times 10^{5} \times 534 \times 415^{2}}{1.15 \times 0.939+(1+0.841 \times 2) \times \frac{0.2+6 \times 0.056}{1+2 \times 0.925}+\frac{0.661 \times 4.5 \times 10^{-4}}{0.87 \times 1.145 \times 10^{-3}}} \\
& =9.77 \times 10^{12} \mathrm{Nmm}^{2}=9.77 \times 10^{3} \mathrm{kNm}^{2} \\
\beta= & \frac{9.77}{6.614}=1.4772, \quad \mu=\frac{1.5}{0.5+\sqrt{1.4772}}=0.874>\text { assumed } 0.8
\end{aligned}
$$

To re-calculate with re-assumed $\mu=0.85$ gives $B_{\text {spl }}=9.78 \times 10^{3}$ and $B_{\text {sul }}=6.615 \times 10^{3} \mathrm{kNm}^{2}, \beta=1.4785$ and $\mu=0.8742$. It can be seen that generally $\mu$ changes a little, so $\mu=0.874$ can be used. From Fig. $5, \gamma=$ 0.148 ( 0.1482 as given in the above from calculation),

$$
\delta_{\max }=0.148 \frac{20.73 \times 5.33^{4}}{24 \times 9.78 \times 10^{3}} \times 10^{3}=10.55 \mathrm{~mm}
$$

\section{DISCUSSION}

Because the adjusting coefficients $\mu$ of support moment given in this paper are established both on the theory and checking of test results, so they are fitted in with the stiffness formulas proposed by the author. From the above calculation it can be seen that $\mu$ doesn't change too much in re-calculation because $\beta$ appears in square root. Hence there arises a question, i. e. , whether the other stiffness formulas can be used for calculation of $\beta$ in Eqs. (3), (4). The answer is definite if other stiffness formulas can also give exacter results in checking deflections, at least, for simply supported beams. Otherwise the other formulas of $\mu$ may be appropriately used. For example the formula of stiffness (effective $E_{c} I_{c}$ ) in ACI Code (318-83) can give exacter calculation for beams with rectangular and $\mathrm{T}$ sections under short-term loading, but for sections with tensile flange, it will give results on too great side as considering this flange and will give too small results as no doing so. These facts can be seen from checking the deflections of the above-mentioned 336 beams among which there are some with tensile flange ${ }^{4}$. Hence for the continuous beams with rectangular section, the ACI formula can also be used for calculation of Eq. ( 3 ), (4).

As for the deflection increase of beams under long-term loading, in ACI Code (318-83), additional deflection is proposed to be calculated from multiplying the short-term one by coefficient $\lambda=\left(2-1.2 \rho^{\prime} / \rho\right)$ $\geqq 0.6$, of which the test basis is dominantly on the earlier tests of slabs and beams loaded at the ages of 6 and 14 days respectively, conducted by G.W. Washa ${ }^{12)}$, in these members the influences of creep and shrinkage on deflections were even more great due to early loading, so produced greater increases of deflection. The average increase coefficient $\left(\delta_{l} / \delta_{s}\right)$ of deflection in the long-term tests of various kinds of beams, started to be loaded about at standard age of 28-day at NIT ${ }^{11)}$, is approximately equal to 2 and should be increase or decreased for inverted $\mathrm{T}$ beams or doubly reinforced beams.

Nevertheless, to use the average stiffness of inverted T section, calculated following ACI Code with and without consideration of the tensile flange, perhaps may be proper. 


\section{CONCLUSION}

In this paper, the theoretical basis to consider the interior support to be approximately fixed in calculation of the deflections of continuous beam and the adjusting coefficients $\mu$ of support moment, derived from new inflection points due to redistribution of internal forces following the above sketches are given. Checking computation of moments calculated from the proposed formula with those measured in tests shows that there exists good accordance.

The proposed short- and long-term stiffness formulas are simple. The comparison with numerous test data shows that the method given here is exact.

The diagrams of deflection coefficients in different $\mu$ for continuous beams, given in this paper, are feasible to be used.

\section{APPENDIX}

\section{On Principle of Min. Stiffness}

The stiffness formula is established on pure bending. In a beam with diagonal cracks occurring, the sections are subjected to oblique bending in diagonally cracked sections and shear although the normal bending moments in shear span are small. A beam subjected to two symmetrical loads is taken for example as shown in Fig. A-1 (a).

Owing to the above facts, the stiffness between concentrated load and the section nearby the support is slightly different (concerned with the development of diagonal cracks) from that of the section with max. bending moment. This is because of that the steel strain is increased due to oblique bending in the diagonal section after the diagonal cracks have occurred, while the stiffness of the uncracked portion near the support is much greater as shown in Fig. A-1 (b). However, because the portion of increased stiffness is near the support the influence on the span deflection of beam is much smaller, because the increase of stiffness is equivalent to reduce the loads of conjugate beam, i. e., to reduce the small shadowed areas in Fig. A-1 (d). For practive, in general, it is allowable to calculate the deflection in terms of min. stiffness of the section with max. moment. The steel stress measured by the author in shear span is shown in Fig. A-2. It can be seen that the steel stress is greater than that determined from the normal bending moment ${ }^{4)}$. Besides, the effect of shear deformations on deflection will become greater after the diagonal cracks have occurred. In a beam with low steel ratio $\rho$, the diagonal crack occurs at a greater distance from support so the influence of oblique bending and shear are smaller. However, the contrary effect results in a

a)

b)

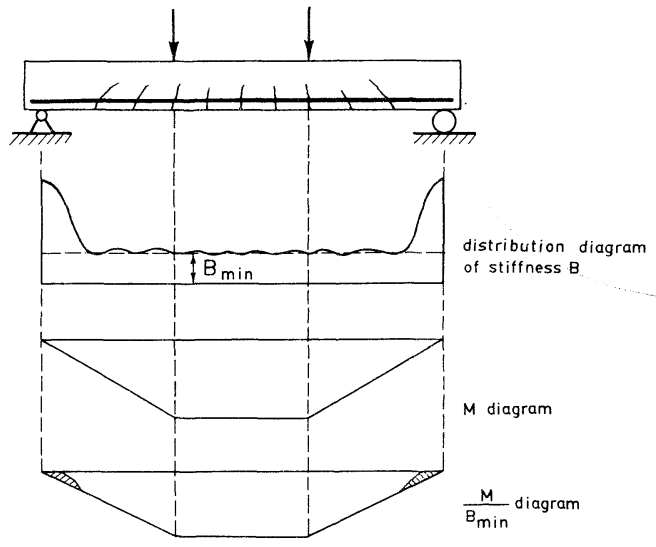

Fig. A.1 Diagram of Stiffness $B$ and $M / B_{\min }$.

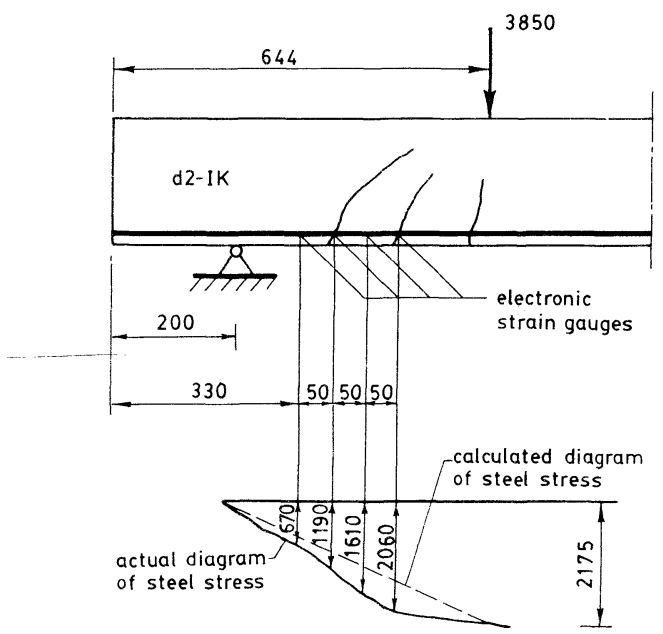

Fig. A.2 Steel stress in shear span. 
beam with high $\rho$. In general case, the errors on great and small sides due to calculating the deflection according to $B_{\min }$ and without considering the influences of oblique bending and shear can be approximately cancelled out each other.

Owing to using a constant stiffness $B_{\min }$ over the span length of a simply supported beam, the deflection formulas given in the course of Strength of Materials or in Handbooks can be used directly. It is equivalent to integrate $M_{x} / B_{\min }$ along beam length doubly. In ACI Code the deflection is also determined in terms of the effective-moment-of-inertia found from max. moment.

If the stiffness formula established on pure bending is used in shear span only in consideration of normal bending, i. e., in consideration of the increase of stiffness with the decrease of normal bending moment, and to integrate numerically along beam length for finding deflection isn't only complicated, but will also give result generally on much smaller side.

In prestressed concrete beam, the diagonal crack occurs at a distance from support greater than that in corresponding non-prestressed beam, so the influences of oblique bending and shear mentioned above is smaller. Hence, to calculate deflections following $B_{\min }$ will give result generally on great side.

As an example, in the tests conducted by the author in the middle of the seventies, the average $\delta_{m} / \delta=$ 0.945 for 55 prestressed members, and average $\delta_{m} / \delta=1.006$ for 16 contrast non-prestress members, where the calculated values of $\delta$ are determined from the average curvatures measured in pure bending length $^{13)}$.

\section{REFERENCES}

1) Handbook for Statical Calculation of Structures (in Chinese), China Building Industry Press (CBIP), Beijing, 1985.

2) Zhang Chong : Study of Redistribution of Elastoplastic Internal Forces in Indeterminate Reinforced Concrete Beams due to Cracks, D. E. Thesis (in Chinese), NIT, April, 1986.

3) Ding Dajun : On the Calculation of Crack Width and Deflection of Reinforced Concrete Beams, Journal of NIT (in Chinese), No. 5, 1964.

4) Ding Dajun, et al. : Stiffness and Crack Width of Reinforced Concrete Members, Journal of Danish Society of Structural Science and Engineering, Vol. 56, No. 4, 1985

5) Ding Dajun and Jiang Yongsheng: Calculation for Complete Curve of Moment in Reinforced Concrete Continuous Beams under Loading, Proceedings of The First Asian Conference on Structural Engineering \& Construction, Bangkok, Jan., 1986.

6) Ding Dajun : Calculations of Deformations and Cracks of Reinforced Concrete Members with Flexure, Selections from Research Papers of Reinforced Concrete Structures (SRPRCS) (in Chinese), CBIP. Beijing, pp. 237 289, 1977.

7) China Design Code of Reinforced Concrete Structures, TJ 10-74 (in Chinese), CBIP, Beijing, 1974.

8) Zhang Chong : Study of the Elastic and Plastic Internal Forces in Reinforced Concrete Continuous Beams, M. E. Thesis, NIT (in Chinese). Dec., 1981.

9) Cao Juyi and Hu Dexin (Academy of Building Research) : Behaviour and Calculation of Redistribution of Internal Forces in Continuous Reinforced Concrete Beams, Research Reports of Redistribution of Plastic Internal Forces in Indeterminate Reinforced Concrete Structures, CBIP (in Chinese), June, 1963.

10) Chen Guomei, et al. : Experimental Study of Limited Values of Moment Adjustment in Continuous Reinforced Concrete Beams (in Chinese), Qinghua University, April, 1981.

11) Ding Dajun, et al. : Experimental Research on Reinforced Concrete and Prestressed Concrete Flexural Members under Long-Term Loading, Journal of Danish Society of Structural Science and Engineering, Vo. 55, No. 3, 1984.

12) Washa, G. W., et al. : Plastic Flow of Reinforced Concrete Continuous Beams, Journal of ACI, No. 1, 1956.

13) Ding Dajun : Experimental Study of Deformations and Cracks of Prestressed Concrete Flexure Members Cracked under Working Load, SRPRCS (in Chinese), Vol. II, CBIP, Beijing, pp. 152 183, 1981.

(Received November 21 1989) 